\title{
Discontinuous Condensation Transition and Nonequivalence of Ensembles in a Zero-Range Process
}

\author{
Stefan Grosskinsky • Gunter M. Schütz
}

Received: 1 October 2008 / Accepted: 15 October 2008 / Published online: 4 October 2008

(C) Springer Science+Business Media, LLC 2008

\section{Erratum to: J Stat Phys (2008) 132: 77-108 \\ DOI 10.1007/s10955-008-9541-z}

Equation (18) in the proof of Proposition 1 contains a computational error, leading to some changes on the same page. The changes do not affect the validity of Proposition 1 , but Proposition 2 and its interpretation have to be restated. Other parts of the manuscript are not affected. In the following we give the corrected version of the proof of Proposition 1, starting with equation (18), and we provide a new proof of the restated Proposition 2 in the appendix:

For $\rho>\rho_{c}$ to leading order

$$
\phi_{R}(\rho) \simeq c_{1}-\left(\frac{c_{1}}{c_{0}}\right)^{R / 2} \frac{c_{1}}{\sqrt{z_{\infty}\left(c_{1}\right)\left(\rho-\rho_{c}\right)}} \rightarrow c_{1} \quad \text { as } L \rightarrow \infty .
$$

For $\rho=\rho_{c}$ the correction has a different power $\left(\frac{c_{1}}{c_{0}}\right)^{R / 4}$ which leads to the same behaviour as for $\rho>\rho_{c}$. Inserting in (9) this yields analogously to (17)

$$
z_{R}\left(\phi_{R}(\rho)\right)=z_{\infty}\left(\phi_{R}(\rho)\right)\left(1+\left(\frac{c_{1}}{c_{0}}\right)^{R / 2} \sqrt{\frac{\rho-\rho_{c}}{z_{\infty}\left(c_{1}\right)}}\right) \rightarrow z_{\infty}\left(c_{1}\right) \quad \text { as } L \rightarrow \infty
$$

so that $v_{\phi_{R}(\rho), R}^{L} \rightarrow v_{c_{1}, \infty}$ weakly.

The online version of the original article can be found under doi:10.1007/s10955-008-9541-z.

S. Grosskinsky ( $₫)$

Mathematics Institute, Zeeman Building, University of Warwick, Coventry CV4 7AL, UK

e-mail: S.W.Grosskinsky@warwick.ac.uk 
Note that by Proposition 1 the density does not converge if $\rho>\rho_{c}$ since

$$
\rho_{R}\left(\phi_{R}(\rho)\right)=\rho \not \supset \rho_{c}=\rho_{\infty}\left(c_{1}\right)
$$

and the variance of $\eta_{x}$ even diverges as

$$
\operatorname{Var}\left(\eta_{x}\right)=\left.\phi \partial_{\phi} \rho_{R}(\phi)\right|_{\phi=\phi_{R}(\rho)} \simeq 2 c_{1}\left(\frac{c_{0}}{c_{1}}\right)^{R / 2-1} .
$$

Therefore there is no standard law of large numbers for the measures $v_{\phi_{R}(\rho), R}^{L}$ when $\rho>\rho_{c}$. In particular one can show the following.

Proposition 2 For each L let $\eta_{1}^{L}, \ldots, \eta_{L}^{L}$ be iid random variables with distribution $v_{\phi_{R}(\rho), R}^{1}$ and assume that $R \gg \log L$. Then

$$
\frac{1}{L} \sum_{x \in \Lambda_{L}} \eta_{x}^{L} \rightarrow\left\{\begin{array}{ll}
\rho, & \rho<\rho_{c} \\
\rho_{c}, & \rho \geq \rho_{c}
\end{array} \quad\right. \text { almost surely. }
$$

Proof See Appendix.

Note that for $\rho>\rho_{c}(20)$ holds due to very large values $\eta_{x} \sim\left(\frac{c_{0}}{c_{1}}\right)^{R / 2}$ having very small probabilities $\left(\frac{c_{1}}{c_{0}}\right)^{R / 2}$, which also leads to divergence of the variance (21). In turn, the small probabilities lead to almost sure convergence of the sample mean to $\rho_{c}<\rho$, which is a nonstandard strong law of large numbers. The breakdown of the standard strong law coincides with the region of nonequivalence of ensembles, as has been observed also in the context of spin systems $[8,31]$.

\section{Appendix}

\section{A.1 Proof of Proposition 2}

For $\rho<\rho_{c}$ (22) follows by standard results and for $\rho>\rho_{c}$ we have as $L \rightarrow \infty$

$$
v_{\phi_{R}(\rho), R}^{1}\left(\eta_{x}^{L}>R\right) \simeq \sqrt{\frac{\rho-\rho_{c}}{z_{\infty}\left(c_{1}\right)}}\left(\frac{c_{1}}{c_{0}}\right)^{R / 2} .
$$

Therefore if we define the truncated occupation numbers $\hat{\eta}_{x}^{L}=\eta_{x}^{L} \wedge R$, we have

$$
\begin{aligned}
& v_{\phi_{R}(\rho), R}^{L}\left(\frac{1}{L} \sum_{x \in \Lambda_{L}}\left(\eta_{x}^{L}-\hat{\eta}_{x}^{L}\right) \neq 0\right)=v_{\phi_{R}(\rho), R}^{L}\left(\text { at least one } \eta_{x}^{L}>R\right) \\
& \quad=1-\left(1-v_{\phi_{R}(\rho), R}^{1}\left(\eta_{x}^{L}>R\right)\right)^{L} \leq C L\left(\frac{c_{1}}{c_{0}}\right)^{R / 2}
\end{aligned}
$$

for some $C>0$ and $L$ large enough. With $R \gg \log L$ this bound is summable in $L$, and the Borel-Cantelli Lemma implies that

$$
\frac{1}{L} \sum_{x \in \Lambda_{L}} \eta_{x}^{L}-\frac{1}{L} \sum_{x \in \Lambda_{L}} \hat{\eta}_{x}^{L} \rightarrow 0 \quad \text { a.s. } \quad \text { as } L \rightarrow \infty .
$$


Moreover $\left\langle\hat{\eta}_{x}\right\rangle=\rho_{c}+O\left(\left(\frac{c_{1}}{c_{0}}\right)^{R / 2}\right)$ and $\operatorname{Var}\left(\hat{\eta}_{x}\right) \leq \frac{c_{0} c_{1}}{\left(c_{0}-c_{1}\right)^{2}}+O\left(R\left(\frac{c_{1}}{c_{0}}\right)^{R / 2}\right)$ and therefore by the usual strong law we have $\sum_{x \in \Lambda_{L}} \hat{\eta}_{x}^{L} \rightarrow \rho_{c}$ a.s.

Taken together, this implies (22) for $\rho>\rho_{c}$, and $\rho=\rho_{c}$ works analogously with the power $R / 2$ replaced by $R / 4$ and a different prefactor in (142). 